\title{
On the generalization of McVittie's model for an inhomogeneity in a cosmological spacetime
}

\author{
Matteo Carrerd* \\ Institute of Physics, University of Freiburg, Hermann-Herder-Strasse 3, D-79104 Freiburg, Germany \\ Domenico Giulini \\ University of Hannover, Appelstrasse 2, D-30167 Hannover, Germany
}

(Dated: August 21, 2009)

\begin{abstract}
McVittie's spacetime is a spherically symmetric solution to Einstein's equation with an energymomentum tensor of a perfect fluid. It describes the external field of a single quasi-isolated object with vanishing electric charge and angular momentum in an environment that asymptotically tends to a Friedmann-Lemaître-Robertson-Walker universe. We critically discuss some recently proposed generalizations of this solution, in which radial matter accretion as well as heat currents are allowed. We clarify the hitherto unexplained constraints between these two generalizing aspects as being due to a geometric property, here called spatial Ricci-isotropy, which forces solutions covered by the McVittie ansatz to be rather special. We also clarify other aspects of these solutions, like whether they include geometries which are in the same conformal equivalence class as the exterior Schwarzschild solution, which leads us to contradict some of the statements in the recent literature.
\end{abstract}

PACS numbers: 98.80.Jk, 04.20.Jb

Contents

\section{Introduction}

\section{The McVittie model}

\section{Geometry of the McVittie ansatz}

A. Relation to conformal Schwarzschild class

B. Spatial Ricci-isotropy

C. Misner-Sharp energy

D. Singularities and trapped surfaces

E. Other global aspects

IV. Attempts to generalize McVittie's model

A. Einstein's equation for the McVittie ansatz

B. Perfect fluid

C. Perfect fluid plus heat flow

D. Perfect fluid plus null fluid

\section{Conclusion}

\section{Acknowledgments}

\section{A. Proof of Proposition 1}

\section{B. Proof of Proposition 2}

\section{Shear-free observer fields in spherically symmetric spacetimes}

\footnotetext{
*Electronic address: matteo.carrera@physik.uni-freiburg.de

${ }^{\dagger}$ Electronic address: domenico.giulini@itp.uni-hannover.de

¥Also at: ZARM, University of Bremen, Am Fallturm

D-28359 Bremen, Germany
}

References
1

2

\section{INTRODUCTION}

Two sets of exact solutions to Einstein's field equation of General Relativity are of paradigmatic importance: The first set describes the gravitational field of quasi-isolated objects in an asymptotically flat spacetime. Among them is the exterior Schwarzschild solution that describes the stationary gravitational field outside a spherically symmetric star or black hole of mass $m$ with vanishing intrinsic angular momentum (spin) and vanishing electric charge. (The latter two features being included in the three-parameter Kerr-Newman family of solutions.) Such asymptotically flat solutions are meant to apply to a region outside the central object which, on one hand, must be sufficiently far from the considered object, so as to legitimately neglect small irregularities of its surface and/or small deviations from perfect spherical symmetry. On the other hand, and more importantly, the region of applicability must also be sufficiently close to the considered object in order not to include, or come close to, other compact sources, or not contain too much dust-filled space between it and the object which would also act as disturbing source for the gravitational field. In particular, the large-distance asymptotic behavior of such solutions is an idealization and not meant to be strictly that of any object in the real world.

On the other hand, the second set of paradigmatic solutions are the cosmological ones, which aim to model the behavior of space-time at the largest cosmological scales, without trying to be realistic at smaller scales. Among them is the family of homogeneous and isotropic Friedmann-Lemaitre-RobertsonWalker (FLRW) cosmologies on which the cosmological 
standard-model is based.

Given that situation, the task is to combine the virtues of both classes of solutions without the corresponding deficiencies. This means to find exact solutions for the gravitational field of a compact object 'immersed' (see below) into an otherwise cosmological background. This would appear to be an easy task if the field equations were linear, for, in that case, one would just add the solution that describes the gravitational field of a compact object in an otherwise empty universe to the cosmological solution that corresponds to a homogeneous distribution of background matter. Here the mathematical operation of addition appears to be the obvious realization of what one might be tempted to call 'simultaneous physical presence' and hence, in view of the individual interpretations of both solutions, the 'immersion' of the compact object into the cosmological background. But this immediate interpretation in physical terms of a simple mathematical operation is deceptive. This becomes obvious in non-linear theories, like General Relativity (GR), where no simple mathematical operation exists that produces a new solution out of two old ones and where the very same physical question may still be asked.

The proper requirement for a mathematical representation of the envisaged physical situation must, first of all, consist in asymptotic conditions which ensure that the sought for solution approximates the given (e.g. Schwarzschild) one for small distances and a particular cosmological one (e.g. FLRW) for large distances. Second, it must specify somehow the physics in the intermediate region. Usually this will include a specification of the matter components and their dynamical laws together with certain initial and boundary conditions. Needless to say that this will generally result in a complex system of partial differential equations. Most analytic approaches therefore impose further simplifying assumptions that automatically guarantee the right asymptotic behavior and at the same time reduce the free functions to a manageable number.

In this paper we will discuss a particular such approach, which is originally due to McVittie [12] and which has been further analyzed and clarified in a series of carefully written papers by Nolan [15, 16, 17]. Our main motivation is that recently McVittie's solution has been severely criticized as not being able at all to model the envisaged situation [3, [6], whereas a family of slightly generalized ones [4], in which some restrictions concerning the motion of matter and the existence of heat flows is lifted, is argued to be free of the alleged problems. The existence of an exact solution to Einstein's equation that models local inhomogeneities is clearly of great importance, for example in estimating reliable upper bounds to the possible influence of global cosmological expansion onto the dynamics and kinematics of local systems [1].

The paper is organized as follows: In Section $\amalg$ we review what we call the McVittie model. We discuss its metric ansatz and what its entails regarding the geometry of spacetime. Then we discuss the assumptions re- garding the motion of the matter and how this, together with Einstein's equation, determines one of the two free functions in the metric ansatz as a simple function of the other. We interpret this condition in terms of an appropriate concept of local mass as saying that the object does not accrete mass from the ambient matter. In Section $\amalg$ we take a second and closer look at the McVittie ansatz and note some of its characteristic features which, we feel, have not sufficiently carefully been taken into account in $[3,4,6]$. In the light of these observations we then discuss in Section IV the attempted generalizations of McVittie's solution in the references just mentioned. We find that some of the conclusions drawn are indeed unwarranted.

\section{THE MCVITTIE MODEL}

The characterization of the McVittie model is made through two sets of a priori specifications. The first set concerns the metric (left side of Einstein's equations) and the second set the matter (right side of Einstein's equations). The former consists in an ansatz for the metric, which can formally be described as follows: Write down the Schwarzschild metric for the mass parameter $m$ in isotropic coordinates, add a conformal factor $a^{2}(t)$ to the spatial part, and allow the mass parameter $m$ to depend on time. Hence the metric reads

$$
\begin{aligned}
\boldsymbol{g} & =\left(\frac{1-m(t) / 2 r}{1+m(t) / 2 r}\right)^{2} \boldsymbol{d} t^{2} \\
& -\left(1+\frac{m(t)}{2 r}\right)^{4} a^{2}(t)\left(\boldsymbol{d} r^{2}+r^{2} \boldsymbol{g}_{S^{2}}\right)
\end{aligned}
$$

where $\boldsymbol{g}_{S^{2}}=\boldsymbol{d} \theta^{2}+\sin ^{2} \theta \boldsymbol{d} \varphi^{2}$ is the standard metric on the unit 2-sphere. Here we restricted attention to the asymptotically spatially flat (i.e. $k=0$ ) FLRW metric, which is compatible with current cosmological data [11]. For simplicity we shall refer to (1) simply as McVittie's ansatz, though this is not quite correct since McVittie started from a general spherically symmetric form and arrived at (1) with $m(t) a(t)=$ const. after imposing a condition that he interpreted as condition for no matterinfall. The ansatz (1) is obviously spherically symmetric with the spheres of constant radius $r$ being the orbits of the rotation group. ${ }^{1}$ In the next section we will discuss in more detail the geometric implications of this ansatz, independent of whether Einstein's equation holds.

\footnotetext{
1 "Spherical symmetry" of a spacetime means the following: There exists an action of the group $S O(3)$ on spacetime by isometries, which is such that the orbits are either two-dimensional and spacelike or fixed points. The "spheres" implicitly referred to in this term correspond to the two-dimensional orbits, even though they might in principle also be two-dimensional real projective spaces. In the cases we discuss here they will be 2 -spheres.
} 
As already discussed in the introduction, the model here is meant to interpolate between the spherically symmetric gravitational field of a compact object and the environment. It is not to be taken too seriously in the region very close to the central object, where the basic assumptions on the behavior of matter definitely turn unphysical. However, as discussed in [1], at radii much larger than (in geometric units) the central mass (to be defined below) the $k=0 \mathrm{McVittie}$ solution seems to provide a viable approximation for the envisaged situation.

The second set of specifications, concerning the matter, is as follows: The matter is a perfect fluid with density $\varrho$ and isotropic pressure $p$. Hence its energy-momentum tensor is given by $^{2}$

$$
\boldsymbol{T}=\varrho \underline{\boldsymbol{u}} \otimes \underline{\boldsymbol{u}}+p(\underline{\boldsymbol{u}} \otimes \underline{\boldsymbol{u}}-\boldsymbol{g}) .
$$

Furthermore, and this is where the two sets of specifications make contact, the motion of the matter (i.e. its four-velocity field) is given by

$$
\boldsymbol{u}=e_{0},
$$

where $\boldsymbol{e}_{0}$ is the normalization of $\boldsymbol{\partial} / \boldsymbol{\partial} t$ (compare (10)). Finally, the explicit cosmological constant on the lefthand side of Einstein's equation is assumed to be zero, which implies no loss of generality, since a non-zero cosmological constant can always be regarded as special part of the matter's energy-momentum tensor (compare IV). No further assumptions are made. In particular, an equation of state, like $p=p(\varrho)$, is not assumed. The reason for this will become clear soon. Later generalizations will mainly concern (2) and (3).

The Einstein equation ${ }^{3}$ now links the specifications of geometry with that of matter. It is equivalent to the following three relations between the four functions $m(t), a(t), \varrho(t, r)$, and $p(t, r)$ :

$$
\begin{aligned}
& (a m) \cdot=0, \\
& 8 \pi \varrho=3\left(\frac{\dot{a}}{a}\right)^{2}, \\
& 8 \pi p=-3\left(\frac{\dot{a}}{a}\right)^{2}-2\left(\frac{\dot{a}}{a}\right) \cdot\left(\frac{1+m / 2 r}{1-m / 2 r}\right) .
\end{aligned}
$$

Note that here Einstein's equation has only three independent components (as opposed to four for a general spherically symmetric metric), which is a consequence of the fact that the Einstein tensor for the McVittie ansatz (1) is spatially isotropic. This will be discussed in more detail in the next section.

\footnotetext{
${ }^{2}$ Here and in what follows we denote the metric-dual (1-form) of a vector $\boldsymbol{u}$ by underlining it, that is, $\underline{\boldsymbol{u}}:=\boldsymbol{g}(\boldsymbol{u}, \cdot)$ is the 1form metric-dual to the vector $\boldsymbol{u}$. In local coordinates we have $\boldsymbol{u}=u^{\mu} \boldsymbol{\partial}_{\mu}$ and $\underline{\boldsymbol{u}}=u_{\mu} \boldsymbol{d} x^{\mu}$, where $u_{\mu}:=g_{\mu \nu} u^{\nu}$.

${ }^{3}$ We speak of "the Einstein equation" in the singular since we think of it as a single tensor equation, which only upon introducing a coordinate system decomposes in many scalar equations.
}

Equation (4a) can be immediately integrated:

$$
m(t)=\frac{m_{0}}{a(t)},
$$

where $m_{0}$ is an integration constant. Below we will show that this integration constant is to be interpreted as the mass of the central body.

Clearly the system (4) is under-determining. This is expected since no equation of state has yet been imposed. The reason why we did not impose such a condition can now be easily inferred from (4): whereas (4b) implies that $\varrho$ only depends on $t$, (4c) implies that $p$ depends on $t$ and $r$ iff $(\dot{a} / a)^{\cdot} \neq 0$. Hence a non-trivial relation $p=p(\varrho)$ is simply incompatible with the assumptions made so far. The only possible ways to specify $p$ are $p=0$ or $\varrho+p=0$. In the first case (4c) implies that $\dot{a} / a=0$ if $m_{0} \neq 0$ (since then the second term on the right-hand side is $r$ dependent, whereas the first is not, so that both must vanish separately), which corresponds to the exterior Schwarzschild solution, or $a(t) \propto t^{2 / 3}$ if $m_{0}=0$, which leads to the flat FLRW solution with dust. In the second case the fluid just acts like a cosmological constant $\Lambda=8 \pi \varrho$ (using the equation of state $\varrho+p=0$ in $\operatorname{div} \boldsymbol{T}=0$ it implies $\boldsymbol{d} p=0$ and this, in turn, using again the equation of state, implies $\boldsymbol{d} \varrho=0$ ) so that this case reduces to the Schwarzschild-deSitter solution. To see this explicitly, notice first that (4b]4c) imply the constancy of $H=\dot{a} / a=\sqrt{\Lambda / 3}$ and hence one has $a(t)=a_{0} \exp (t \sqrt{\Lambda / 3})$. With such a scalefactor the McVittie metric (10) with (5) turns into the Schwarzschild-de Sitter metric in spatially isotropic coordinates. The explicit formulae for the coordinate transformation which brings the latter in the familiar form can be found in Section 5 of [18] and also in Section 7 of [10]. Finally, note from (4a) that constancy of one of the functions $m$ and $a$ implies constancy of the other. In this case (4b]4C) imply $p=\varrho=0$, so that we are dealing with the exterior Schwarzschild spacetime.

A specific McVittie solution can be obtained by choosing a function $a(t)$, corresponding to the scale function of the FLRW spacetime which the McVittie model is required to approach at spatial infinity, and the constant $m_{0}$, corresponding to the 'central mass'. Relations (4b/4c), and (5) are then used to determine $\varrho, p$, and $m$, respectively. Clearly this 'poor man's way' to solve Einstein's equation holds the danger of arriving at unrealistic spacetime dependent relations between $\varrho$ and $p$. This must be kept in mind when proceeding in this fashion. For further discussion of this point we refer to [15, 16].

As will be discussed in more detail in Section $\amalg I C$ below, in the spherically-symmetric case the concept of local mass (or energy) is well captured by the Misner-Sharp (MS) energy [13], whose purely geometric definition in terms of Riemannian curvature allows to decompose it into a sum of two terms, one of which comes from the Ricci- the other from the Weyl curvature. It is the latter which may be identified with the gravitational mass of 
the central object. Applied to (1), the Weyl contribution to the MS energy can be written in the following form, also taking into account (5),

$$
\begin{aligned}
& E_{\mathrm{R}}=\frac{4 \pi}{3} R^{3} \varrho, \\
& E_{\mathrm{W}}=m_{0} .
\end{aligned}
$$

The constancy of $E_{\mathrm{w}}$ is then interpreted as saying that no energy is accreted from the ambient matter onto the central object.

We now briefly discuss the basic properties of the motion of cosmological matter. Being spherically symmetric, the velocity field $\boldsymbol{u}$ specified in (3) is automatically vorticity free. The last property is manifest from its hypersurface orthogonality, which is immediate from (1). Moreover, $\boldsymbol{u}$ is also shear free. This, too, can be immediately read off (11) once one takes into account the following result, whose proof we sketch in Appendix C A spherically symmetric normalized timelike vector field $\boldsymbol{u}$ in a spherically symmetric spacetime $(\mathcal{M}, \boldsymbol{g})$ is shear free iff its corresponding spatial metric, that is, the metric $\boldsymbol{g}$ restricted to the subbundle $\boldsymbol{u}^{\perp}:=\{\boldsymbol{v} \in T \mathcal{M} \mid \boldsymbol{g}(\boldsymbol{v}, \boldsymbol{u})=$ $0\}$, is conformally flat. The metric (1) obviously is spatially conformally flat with respect to the choice (3) made here. Moreover, the expansion (divergence) of $\boldsymbol{u}$ is

$$
\theta=3 H
$$

where $H:=\dot{a} / a$, just as in the FLRW case. In particular, the expansion of the cosmological fluid is homogeneous in space. Exactly as in the FLRW case is also the expression for the variation of the areal radius along the integral lines of $\boldsymbol{u}$ (that is the velocity of cosmological matter measured in terms of its proper time and the areal radius):

$$
\boldsymbol{u}(R)=H R
$$

which is nothing but Hubble's law. Recall that for a spherically symmetric spacetime the areal radius, denoted here by $R$, is the function defined by $R(p):=$ $\sqrt{\mathcal{A}(p) / 4 \pi}$, where $\mathcal{A}(p)$ is the proper area of the 2dimensional $S O(3)$-orbit through the point $p$. For the McVittie spacetime the areal radius is given explicitly in (13). The acceleration of $\boldsymbol{u}$, which in contrast to the FLRW case does not vanish here, is given by

$$
\boldsymbol{\nabla}_{\boldsymbol{u}} \boldsymbol{u}=\frac{m_{0}}{R^{2}}\left(\frac{1+m / 2 r}{1-m / 2 r}\right) \boldsymbol{e}_{1}
$$

Here $\boldsymbol{e}_{1}$ is the normalized vector field in radial direction as defined in (10). In leading order in $m_{0} / R$ this corresponds to the acceleration of the observers moving along the timelike Killing field in Schwarzschild spacetime.

It is also important to note that the central gravitational mass in McVittie's spacetime may be modeled by a shear-free perfect-fluid star of positive homogeneous energy density [14]. The matching is performed along a world-tube comoving with the cosmological fluid, across which the energy density jumps discontinuously. This means that the star's surface is comoving with the cosmological fluid and hence, in view of (7), that it geometrically expands (or contracts). This feature, however, should be merely seen as an artifact of the McVittie model (in which the relation (7) holds), rather than a general property of compact objects in any cosmological spacetimes. Positive pressure within the star seems to be only possible if $2 a \ddot{a}+\dot{a}^{2}<0$ (see Eq. (3.27) in [14] with $a=\exp (\beta / 2))$, that is, for deceleration parameters $q>1 / 2$.

\section{GEOMETRY OF THE MCVITTIE ANSATZ}

In this section we will discuss the geometry of the metric (11) independent of the later restriction that it will have to satisfy Einstein's equation for some reasonable energy-momentum tensor. This means that at this point we shall not assume any relation between the two functions $m(t)$ and $a(t)$, apart from the first being non negative and the second being strictly positive. We will discuss the metric's 'spatial Ricci-isotropy' (a term explained below), its singularities and trapped regions, and also compute its Misner-Sharp energy decomposed into the Ricci and Weyl parts. We shall start, however, by answering the question of what the overlap is between the geometries represented by (11) and the conformal equivalence class of the exterior Schwarzschild geometry.

\section{A. Relation to conformal Schwarzschild class}

This question is an obvious one in view of the way in which (1) is obtained from the exterior Schwarzschild metric. It is clear that for $m=m_{0}=$ const. the metric (1) is conformally equivalent to the exterior Schwarzschild metric, since upon using a new time coordinate $T$ with $d T=d t / a(t)$ we can pull out $a^{2}(t)$ as a common conformal factor. The following proposition, whose proof we shall give in Appendix A, states that a constant $m$ is in fact also necessary condition:

Proposition 1. Let $\mathcal{S}_{\mathrm{McV}}$ denote the set of metrics in the form of the McVittie ansatz (11) (parametrized by the two positive functions $a$ and $m$ ) and $\mathcal{S}_{\mathrm{cS}}$ the set of metrics conformally equivalent to an exterior Schwarzschild metric (parametrized by a positive conformal factor and a constant positive Schwarzschild mass $M_{0}$ ). Then the intersection between $\mathcal{S}_{\mathrm{McV}}$ and $\mathcal{S}_{\mathrm{cS}}$ is given by the subset of metrics in $\mathcal{S}_{\mathrm{McV}}$ with constant $m$ or, equivalently, by the subset of metrics in $\mathcal{S}_{\mathrm{cS}}$ whose conformal factor has a gradient proportional to the Killing field $\boldsymbol{\partial} / \partial T$ of the Schwarzschild metric (see (A1b) for notation).

Note that we excluded the 'trivial' cases in which $m$ or $M_{0}$ (or both) vanish for the following reason: Comparing the expressions for the Weyl part of the MS energy of the two types of metrics (see A10 in Appendix A it 
follows that $m$ vanishes iff $M_{0}$ does and this, in turn, leads to a metric conformally related to the Minkowski metric where the conformal factor depends only on time, that is, a FLRW metric. But such a spacetime, being homogeneous, is not of interest to us here.

In particular, Proposition 1 implies that the metric of Sultana and Dyer [19] are not of type (11), as suggested in Section IV A of [4] and allegedly shown in Section II of [2] (cf. our footnote 5 at page 8). This immediately follows from the observation that the conformal factor, expressed as function of the standard Schwarzschild coordinates that appear in (A1b), is given by $\Omega(T, R)=$ $\left(T+2 M_{0} \ln \left(R / 2 M_{0}-1\right)\right)^{2}$ (compare Eqs. (8) and (9) of [19]), which also depends on $R$ and hence does not satisfy the condition of Proposition 1 We will have to say more about this at the beginning of Section[IV and in SectionIVD,

\section{B. Spatial Ricci-isotropy}

An important feature of any metric that is covered by the ansatz (11) is, that its Einstein tensor is spatially isotropic in the following sense: 'Spatially' refers to the directions orthogonal to $\boldsymbol{\partial} / \boldsymbol{\partial} t$ and 'isotropy' to the condition that the spatial restriction of the spacetime's Einstein tensor is proportional to the spatial restriction of the metric. Note that, since the spacetime's metric is time dependent, the spatial restriction of the spacetime's Einstein or Ricci tensor is not the same as the Einstein or Ricci tensor of the spatial sections with their induced metrics. Hence the notion of spatial isotropy of the Einstein tensor used here is not the same as saying that the induced metric of the slices is an Einstein metric.

Given that the Einstein tensor of (10) is spatially isotropic in the sense used here, it is then obvious that Einstein's equation will impose a severe restriction upon the matter's energy-momentum tensor, saying that it, too, must be spatially isotropic. The degree of specialization implied by this will be discussed in more detail below. Here we only remark that this observation already answers in the negative a question addressed, and left open, in the last paragraph of [3] , of whether (1) is the most general spherically symmetric solution describing a black hole embedded in a spatially flat FLRW background: It clearly is not.

In passing we make the obvious remark that, since Ein $=$ Ric $-(1 / 2)$ Scal $\boldsymbol{g}$, where Ric denotes the Ricci tensor, the Einstein tensor is spatially isotropic iff the same holds for the Ricci tensor. For this reason we will from now on refer to spatial Ricci-isotropy to denote the feature in question.

Now, a way to actually show spatial Ricci-isotropy is to compute the components of the Einstein tensor with respect to the orthonormal tetrad $\left\{\boldsymbol{e}_{\mu}\right\}_{\mu \in\{0, \cdots, 3\}}$ of (1) defined by

$$
\boldsymbol{e}_{\mu}:=\left\|\boldsymbol{\partial} / \boldsymbol{\partial} x^{\mu}\right\|^{-1} \boldsymbol{\partial} / \boldsymbol{\partial} x^{\mu}
$$

where $\left\{x^{\mu}\right\}=\{t, r, \theta, \varphi\}$. Here, and henceforth, we write $\|\boldsymbol{v}\|:=\sqrt{|\boldsymbol{g}(\boldsymbol{v}, \boldsymbol{v})|}$. Note that $\boldsymbol{e}_{0}, \boldsymbol{e}_{1}$ are orthogonal to and $\boldsymbol{e}_{2}, \boldsymbol{e}_{3}$ tangent to the 2 -spheres of constant radius $r$. The non-vanishing independent components of the Einstein tensor with respect to the orthonormal basis (10) are:

$$
\begin{aligned}
& \operatorname{Ein}\left(\boldsymbol{e}_{0}, \boldsymbol{e}_{0}\right)=3 F^{2}, \\
& \operatorname{Ein}\left(\boldsymbol{e}_{0}, \boldsymbol{e}_{1}\right)=\frac{2}{R^{2}}\left(\frac{A}{B}\right)^{2}(a m), \\
& \operatorname{Ein}\left(\boldsymbol{e}_{i}, \boldsymbol{e}_{j}\right)=-\left(3 F^{2}+2 \frac{A}{B} \dot{F}\right) \delta_{i j},
\end{aligned}
$$

where an overdot denotes differentiation along $\boldsymbol{\partial} / \boldsymbol{\partial} t$. Before explaining the functions $A, B, R$, and $F$, note that the spatial isotropy of the Einstein tensor follows immediately from (11c), since $\operatorname{Ein}\left(\boldsymbol{e}_{i}, \boldsymbol{e}_{j}\right) \propto \delta_{i j}$. In (11) and in the following we set:

$$
A(t, r):=1+m(t) / 2 r, \quad B(t, r):=1-m(t) / 2 r,
$$

and

$$
R(t, r)=\left(1+\frac{m(t)}{2 r}\right)^{2} a(t) r,
$$

where $R$ is the areal radius for the McVittie ansatz (11), and also

$$
F:=\frac{\dot{a}}{a}+\frac{1}{r B} \frac{(a m)^{\cdot}}{a} .
$$

In passing we note that both quantities, $F$ and $a m$, that appear in the components of the Einstein tensor, have a geometrical interpretation: the former is one third the expansion of the vector field $\boldsymbol{e}_{0}$, that is, $F=\operatorname{div}\left(\boldsymbol{e}_{0}\right) / 3$, and the latter is the Weyl part of the Misner-Sharp energy of the metric (11) (see (22), below). Moreover, as we already noted in Section II, the observer field $\boldsymbol{e}_{0}$ is free of vorticity and shear. Hence, taking into account the relation (C5) between the expansion $\theta$ and the shear scalar $\sigma$ of an arbitrary spherically-symmetric observer field, the expansion of $\boldsymbol{e}_{0}$ can be simply written as $3 \boldsymbol{d} R\left(\boldsymbol{e}_{0}\right) / R$ so that $F$ may be expressed as

$$
F=\boldsymbol{d} R\left(\boldsymbol{e}_{0}\right) / R \text {. }
$$

In order to estimate the degree of specialization implied by spatial Ricci-isotropy, we ask for the most general spherically symmetric metric for which this is the case. To answer this, we first note that any spherically symmetric metric can always be written in the form

$$
\boldsymbol{g}=\left(\frac{B(t, r)}{A(t, r)}\right)^{2} \boldsymbol{d} t^{2}-a^{2}(t) A^{4}(t, r)\left(\boldsymbol{d} r^{2}+r^{2} \boldsymbol{g}_{S^{2}}\right)
$$

This reduces to McVittie's ansatz (1) if $A, B$ are given by (12). For the general spherically symmetric metric (16), spatial Ricci-isotropy can be shown to be equivalent to

$$
\delta^{2}(A B)-8(\delta A)(\delta B)=0,
$$

where $\delta:=r^{-1} \partial / \partial r=2 \partial / \partial r^{2}$. It is obvious that there are many more solutions to this differential equation than just (12). 


\section{Misner-Sharp energy}

In order to be able to interpret (1) as an ansatz for an inhomogeneity in a FLRW universe, it is useful to compute the Misner-Sharp (MS) energy and, in particular, its Ricci and Weyl parts. This concept of quasi-local mass, which is defined only for spherically symmetric spacetimes, and which in this case coincides with Hawking's more general definition [8] of quasi-local mass (see e.g. 1]), allows to detect localized sources of gravity. 13]:

We recall the geometric definition of the MS energy 9 ,

$$
E:=-\frac{1}{2} R^{3} K,
$$

where $R$ denotes the areal radius and $K$ the extrinsic curvature. More precisely, the equation should be read and understood as follows: First of all, the quantities $R$ and $K$, and hence also $E$, are real-valued functions on spacetime. In order to determine their values at a point $p$, recall that, due to the requirement of spherical symmetry, there is a unique two-(or zero-) dimensional $S O(3)$ orbit $S(p)$ through $p$. The value of $R$ at $p$ is as explained below Eq. (8) and the value of $K$ at $p$ is

$$
K(p):=\frac{\boldsymbol{R i e m}\left(\boldsymbol{X}_{p}, \boldsymbol{Y}_{p}, \boldsymbol{X}_{p}, \boldsymbol{Y}_{p}\right)}{\boldsymbol{g}\left(\boldsymbol{X}_{p}, \boldsymbol{X}_{p}\right) \boldsymbol{g}\left(\boldsymbol{Y}_{p}, \boldsymbol{Y}_{p}\right)-\left(\boldsymbol{g}\left(\boldsymbol{X}_{p}, \boldsymbol{Y}_{p}\right)\right)^{2}} .
$$

Here Riem is the (totally covariant) Riemannian curvature tensor of spacetime and $\boldsymbol{X}_{p}$ and $\boldsymbol{Y}_{p}$ are any two linearly independent vectors in the tangent space at $p$ which are also tangent to the orbit $S(p)$. Note that the right-hand side only depends of the plane spanned by $\boldsymbol{X}_{p}, \boldsymbol{Y}_{p}$ and not on the vectors spanning it. Finally we note that the minus sign in (18) is just a relict of our signature choice (mostly minus).

From the curvature decomposition for a spherically symmetric metric (see [1]) one can rewrite (18) in the form

$$
E=\frac{R}{2}(1+\boldsymbol{g}(\nabla R, \nabla R)),
$$

where $\nabla R$ denotes the gradient vector-field of $R$. This provides a convenient expression for the computation of the MS energy. For a self-contained review of the basic properties of the MS energy as well as its interpretation as the amount of active gravitational energy contained in the interior of the spheres of symmetry ( $S O(3)$-orbits) and its relation with the other mass concepts, see [1].

The decomposition of the Riemann tensor into a Ricci and a Weyl part leads, together with (18), to a natural decomposition of the MS energy into a Ricci and Weyl part (see also [1]). For the Ricci part of the MS energy of (1) we get

$$
E_{\mathrm{R}}=\frac{1}{6} R^{3} \operatorname{Ein}\left(\boldsymbol{e}_{0}, \boldsymbol{e}_{0}\right)=\frac{R}{2}\left(\boldsymbol{d} R\left(\boldsymbol{e}_{0}\right)\right)^{2} .
$$

The first equality in (21) can be derived by merely using the spatial Ricci-isotropy in the expression for the Ricci part of the Riemann tensor. The second equality follows then with (11a) and (15). The Weyl part can now be obtained as the difference between the full MS energy and (21). We use the expression (20) for the former and write $\boldsymbol{g}(\boldsymbol{\nabla} R, \boldsymbol{\nabla} R)=\left(\boldsymbol{e}_{0}(R)\right)^{2}-\left(\boldsymbol{e}_{1}(R)\right)^{2}$. The part involving $\boldsymbol{e}_{0}(R)$ equals the Ricci part (21) and hence the Weyl part is given by $(R / 2)\left(1-\left(\boldsymbol{e}_{1}(R)\right)^{2}\right)$. From (13) we calculate $\boldsymbol{e}_{1}(R)$ and hence obtain for the Weyl part of the MS energy:

$$
E_{\mathrm{w}}=a m .
$$

The Ricci part of the MS energy is that part which, via Einstein's equation, can be locally related to the matter's energy-momentum tensor, whereas the gravitational mass of the central object is contained in the Weyl part of the MS energy. Notice that the latter is spatially constant (the functions $a$ and $m$ in (22) only depend on time) but may depend on time. If the latter is the case we interpret this as saying that the central mass exchanges energy with the ambient matter.

\section{Singularities and trapped surfaces}

Next we comment on the singularity properties of the McVittie ansatz (11). From (11c) one suspect, because of the term proportional to $1 / B$, a singularity in the Ricci part of the curvature at $r=m / 2$ (that is at $R=2 a m=$ $\left.2 E_{\mathrm{w}}\right)$. In fact, this corresponds to a genuine curvature singularity, as one can see from looking, for example, at the following expression for the scalar curvature (i.e. the Ricci scalar),

$$
\text { Scal }=-12 F^{2}-6 \frac{A}{B} \dot{F},
$$

which can be quickly computed from (11). In Appendix $B$ we insert into this expression the definition (14) of $F$ and expand this in powers of $1 /(r B)$. This allows to prove

Proposition 2. The Ricci scalar for a metric of the form (11) becomes singular in the limit $r \rightarrow m / 2$ for any functions a and $m$, except for the following three special cases:

(i) $m=0$ and a arbitrary (FLRW),

(ii) $a$ and $m$ are constant (Schwarzschild), and

(iii) $(a m)^{\cdot}=0$ and $(\dot{a} / a)^{\circ}=0$ (Schwarzschild-de Sitter).

This means that, as long as we stick to the ansatz (1), at $r=m / 2$ there will always (with the only exceptions listed above) be a singularity in the Ricci part of the curvature and thus, assuming Einstein's equation is satisfied, also in the energy momentum tensor, irrespectively of the details of the underlying matter model. Hence any attempt to eliminate this singularity by maintaining the ansatz (1) and merely modifying the matter model is doomed to fail.

In particular, this is true for the generalizations presented in [4], contrary to what is claimed in that work and its follow ups $[3,[6]$. We also remark that it makes no 
sense to absorb the singular factors $1 / B$ in front of the time derivatives by writing $(A / B) \boldsymbol{\partial} / \boldsymbol{\partial} t$ as $\boldsymbol{e}_{0}$ and then argue, as was done in [4], that this eliminates the singularity. The point is simply that then $e_{0}$ applied to any continuously differentiable function diverges as $r \rightarrow m / 2$. Below we will argue that this singularity lies within a trapped region.

Specializing to the McVittie model, recall that in this case it is assumed that the fluid moves along the integral curves of $\boldsymbol{\partial} / \boldsymbol{\partial} t$, which become lightlike in the limit as $r$ tends to $m / 2$. Their acceleration is given by the gradient of the pressure, which necessarily diverges in the limit $r \rightarrow m / 2$, as one explicitly sees from (9). For a more detailed study of the geometric singularity at $r=m / 2$, see [16, 17].

For spherically symmetric spacetimes the Weyl part of the curvature has only a single independent component, which is $-2 / R^{3}$ times the Weyl part of the MS energy, by the very definition of the latter (see [1]). The square of the Weyl tensor for the ansatz (1) may then be conveniently expressed as

$$
\langle\text { Weyl, Weyl }\rangle=48 \frac{(a m)^{2}}{R^{6}}
$$

This shows that $R=0$ also corresponds to a genuine curvature singularity, though this is not part of the region covered by our original coordinate system, for which $r>$ $m / 2$ (that is $R>2 E_{\mathrm{W}}$ ).

It is instructive to also determine the trapped regions of McVittie spacetime. Recall that a spacelike 2-sphere $S$ is said to be trapped, marginally trapped, or untrapped if the product $\theta^{+} \theta^{-}$of the expansions (for the definitions see e.g. [1]) for the ingoing and outgoing futurepointing null vector fields normal to $S$ is positive, zero, or negative, respectively. Taking $S$ to be $S_{R}$, that is, an $S O(3)$ orbit with areal radius $R$, it immediately follows from the relation $2 \theta^{+} \theta^{-}=\boldsymbol{g}(\boldsymbol{\nabla} R, \boldsymbol{\nabla} R) / R^{2}$ (see [1] ) that $S_{R}$ is trapped, marginally trapped, or untrapped iff $\boldsymbol{g}(\boldsymbol{\nabla} R, \boldsymbol{\nabla} R)$ is positive, zero, or negative, respectively. This corresponds to timelike, lightlike, or spacelike $\boldsymbol{d} R$, or equivalently, in view of (20), to $2 E-R$ being positive, zero, or negative, respectively. Using (21) together with (11a), the MS energy for the McVittie ansatz can be written as $E=E_{\mathrm{w}}+R^{3} F^{2} / 2$, so that

$$
2 E-R=F^{2} R^{3}-R+R_{S} .
$$

Here we defined the 'Schwarzschild radius' as $R_{S}:=2 E_{\mathrm{w}}$, which generally will depend on time. We wish to determine the values of the radial coordinate $(r$ or $R$ ) at which the expression (25) assumes the value zero. We shall continue to work with $R$ rather than $r$ since $R$ has the proper geometric meaning of areal radius. In the region we are considering (that is $r>m / 2$ or, equivalently, $R>R_{S}$ ) the inversion of (13) reads $r(R)=$ $R\left(1-R_{S} / 2 R+\sqrt{1-R_{S} / R}\right) / 2 a$, so that (25) divided by $R_{S}$ can be written in the form

$$
\frac{2 E-R}{R_{S}}=\left(\eta+\frac{\varepsilon}{x-1+\sqrt{x(x-1)}}\right)^{2} x^{3}-x+1 .
$$

Here we introduced the dimensionless radial coordinate $x:=R / R_{S}$ and the (small) parameters $\varepsilon:=\dot{R}_{S}$ and $\eta:=R_{S} / R_{H}$, where $R_{H}:=1 / H$ denotes the 'Hubble radius'. Recall that since $R>R_{S}$ we have $x>1$.

Consider first the McVittie case, in which $\varepsilon=\dot{R}_{S}=0$. Then (26) turns into a cubic polynomial in $x$ which is positive for $x=0$ and tends to $\pm \infty$ for $x \rightarrow \pm \infty$. Hence it always has a negative zero (which does not interest us) and two positive zeros iff

$$
R_{S} / R_{H}<2 / 3 \sqrt{3} \approx 0.38 .
$$

This clearly corresponds to the physical relevant case where the Schwarzschild radius is much smaller than the Hubble radius. One zero lies in the vicinity of the Schwarzschild radius and one in the vicinity of the Hubble radius, corresponding to two marginally trapped spheres. The exact expressions for the zeros can be easily written down, but are not very illuminating. In leading order in the small parameter $\eta=R_{S} / R_{H}$, they are approximated by

$$
\begin{aligned}
& R_{1}=R_{S}\left(1+\eta^{2}+O\left(\eta^{4}\right)\right), \\
& R_{2}=R_{H}\left(1-\eta / 2+O\left(\eta^{2}\right)\right) .
\end{aligned}
$$

From this one sees that for the McVittie ansatz the radius of the marginally trapped sphere of Schwarzschild spacetime $\left(R_{S}\right)$ increases and that of the FLRW spacetime $\left(R_{H}\right)$ decreases. The first feature can, for the McVittie model, be understood as an effect of the cosmological environment, whereas the latter is an effect of the inhomogeneity in form of a central mass abundance. All the spheres with $R<R_{1}$ or $R>R_{2}$ are trapped and those with $R_{1}<R<R_{2}$ are untrapped. In particular, the singularity $r=m / 2$, that is $R=2 E_{\mathrm{W}}=R_{S}$, lies within the inner trapped region.

In the case in which $\varepsilon=\dot{R}_{S}$ is non-zero and 'small' (see below in which sense), we expect that the zeros (28) vary smoothly in $\varepsilon$ so that, in particular, the singularity at $R=R_{S}$ still remains within the inner trapped region. An expansion in $\varepsilon$ gives, for the zero in the vicinity of the Schwarzschild radius:

$$
R_{1}(\varepsilon)=R_{S}\left(1+\eta^{2}+\left(2-2 \eta+13 \eta^{2}\right) \varepsilon+O\left(\eta^{3}, \varepsilon^{2}\right)\right),
$$

which clearly reduces to (28a) for $\varepsilon=0$. From this expression one sees that, according to the physical expectation, in case of accretion $(\varepsilon>0)$ the inner marginally trapped sphere becomes larger in area, whereas in the opposite case $(\varepsilon<0)$ it shrinks. In our approximation (29), the singularity $R=R_{S}$ continues to lie inside the trapped region for 'accretion rates' $\varepsilon=\dot{R}_{S}>-\eta^{2} / 2$ or, in terms of physical quantities and re-introducing the factors of $c$, 
for $\dot{R}_{S} / c>-\left(R_{S} / R_{H}\right)^{2} / 2$. However, this also characterizes the region of validity of the expansion (29): Given a positive $\eta$, an expansion in $\varepsilon$ around zero exists only for $\varepsilon>-\eta^{2} / 2$ since there exists no expansion on both $(\varepsilon, \eta)$ around $(0,0)$ (this is because the partial derivative of (26) with respect to $x$ does not exist at $x=1$ ).

\section{E. Other global aspects}

Another aspect concerns the global behavior of the McVittie ansatz (1). We note that each hypersurface of constant time $t$ is a complete Riemannian manifold, which, besides the rotational symmetry, admits a discrete isometry given in $(r, \theta, \varphi)$ coordinates by

$$
\phi(r, \theta, \varphi)=\left((m / 2)^{2} r^{-1}, \theta, \varphi\right) .
$$

This corresponds to an inversion at the 2-sphere $r=$ $m / 2$, which shows that the hypersurfaces of constant $t$ can be thought of as two isometric asymptotically-flat pieces joined together at the 2-sphere $r=m / 2$. This 2sphere is totally geodesic since it is a fixed-point set of an isometry; in particular, it is a minimal surface. Except for the time-dependent factor $m(t)$, this is just like for the slices of constant Killing time in the Schwarzschild metric (the difference being that (30) does not extend to an isometry of the spacetime metric unless $\dot{m}=0$ ). Now, the fact that $r \rightarrow 0$ corresponds to an asymptotically flat end of each of the 3 -manifolds $t=$ const. implies that the McVittie metric cannot literally be interpreted as corresponding to a point particle sitting at $r=0(r=0$ is in infinite metric distance) in an otherwise spatially flat FLRW universe, just like the Schwarzschild metric does not correspond to a point particle sitting at $r=0$ in Minkowski space. Unfortunately, McVittie seems to have interpreted his solution in this fashion [12] which even until recently gave rise to some confusion in the literature (e.g. [5, [7, 20]). A clarification was given in [16].

\section{ATTEMPTS TO GENERALIZE MCVITTIE'S MODEL}

The first obvious generalization consists in allowing for a non-vanishing cosmological constant. However, as was already indicated before, this is rather trivial since it merely corresponds to the substitutions $\varrho \rightarrow \varrho+\varrho_{\Lambda}$ and $p \rightarrow p+p_{\Lambda}$ in (4), where $\varrho_{\Lambda}:=\Lambda / 8 \pi$ and $p_{\Lambda}:=-\Lambda / 8 \pi$ are the energy-density and pressure associated to the cosmological constant $\Lambda$.

The attempts to non-trivially generalize the McVittie solution have focused so far on keeping the ansatz (10) and relaxing the conditions on the matter in various ways. In [4] generalization were presented allowing radial fluid motions relative to the observer vector field $\boldsymbol{\partial} / \boldsymbol{\partial} t$ (that is relaxing condition (31) ) as well as including heat conduction. Below we will critically review these attempts, taking due care of the geometric constraints imposed by the ansatz (1), and also outline how to explicitly construct the respective solutions.

Another exact solution that models an inhomogeneity in a cosmological spacetime was presented in [19] by Sultana and Dyer and was recently analyzed in [2]. Here the metric is conformally equivalent to the exterior Schwarzschild metric and the cosmological matter is composed of two non-interacting perfect fluids, one being pressureless dust, the other being a null fluid. One might ask if this solution fits into the class of McVittie models, as was suggested in [4] ${ }^{4}$ and allegedly confirmed explicitly in [2] ${ }^{5}$. However, as we already noted at the end of Section III above in view of Proposition! this is not the case. Two further way to see this are as follows: First, the Sultana-Dyer metric is not spatially Ricci-isotropic ${ }^{6}$ and, second, the McVittie metric is not compatible with the matter model used by Sultana and Dyer, with the sole exception of trivial or exotic cases, as will be shown in SectionIVD below.

\footnotetext{
${ }^{4}$ In Section IV A of [4] it is suggested that the Sultana-Dyer metric is equal to the McVittie metric (1) in which $a(t)=a_{0} t^{2 / 3}$ and $m(t)=m_{0}$, for some constants $a_{0}$ and $m_{0}$ (see Eq. (62) in [4]). Let denote the latter metric by $\tilde{\boldsymbol{g}}$. Indeed, since $m$ is constant and in view of Proposition [1 $\tilde{\boldsymbol{g}}$ is conformally related to the Schwarzschild metric. Moreover, as one may explicitly check via our Eq. 11c), the Einstein tensor of $\tilde{\boldsymbol{g}}$ has a vanishing spherical part. Despite sharing these two properties, $\tilde{\boldsymbol{g}}$ and the SultanaDyer metric are not equal.

5 The problem with the reasoning in Section II of [2] is the following (numbers refer to equations in [2]): It is true by construction that the Sultana-Dyer metric (2.1) is conformally related to the Schwarzschild metric, as expressed in the second line of (2.3) [the first line in (2.3) does not follow], but the conformal function $a$ depends non-trivially on the Schwarzschild coordinates for time and radius (denoted by $\bar{\eta}$ and $\tilde{r}$ in [2]: Cf. our discussion in the last paragraph of Section IIIA. Hence it is not possible to introduce a new time coordinate $\bar{t}$ that satisfies $d \bar{t}=a d \bar{\eta}$ (the right hand side is not a closed 1-form), as pretended in the transition to (2.5).

6 To show this, one has to show that there exists no timelike direction with respect to which the Ricci tensor (or, equivalently, the Einstein tensor) is spatially isotropic. This can be shown as follows: First note that the Einstein tensor of the SultanaDyer metric has the form Ein $=\mu \underline{\boldsymbol{u}} \otimes \underline{\boldsymbol{u}}+\tau \underline{\boldsymbol{k}} \otimes \underline{\boldsymbol{k}}$ (see [19]), where $\boldsymbol{u}$ is a normalized future-pointing spherically-symmetric timelike vector field and $\boldsymbol{k}$ the in-going future-pointing lightlike vector field orthogonal to the $S O(3)$-orbits normalized such that $\boldsymbol{g}(\boldsymbol{u}, \boldsymbol{k})=1$. In particular, the spherical part of the Einstein tensor vanishes: Hence, the Einstein tensor is spatially isotropic iff there exists a non-vanishing spacelike sphericallysymmetric (i.e. orthogonal to the $S O(3)$-orbits) vector field $\boldsymbol{s}$ with $\operatorname{Ein}(\boldsymbol{s}, \boldsymbol{s})=0$. Without loss of generality one can chose $\boldsymbol{s}$ to be normalized: $\boldsymbol{s}=\sinh \chi \boldsymbol{u}+\cosh \chi \boldsymbol{e}$, where $\boldsymbol{e}$ is the normalized vector field orthogonal to $\boldsymbol{u}$ and to the $S O(3)$-orbits pointing in positive radial direction. Hence one has $\boldsymbol{k}=\boldsymbol{u}-\boldsymbol{e}$ and thus: $\operatorname{Ein}(\boldsymbol{s}, \boldsymbol{s})=\mu \sinh ^{2}(\chi)+\tau \exp (2 \chi)$. Clearly, the latter expression vanishes nowhere in the physically interesting region (cf. Eq. (26)
} in [19]), where both $\mu$ and $\tau$ are positive. 


\section{A. Einstein's equation for the McVittie ansatz}

In the following we will restrict to those generalizations of the McVittie model which keep the metric ansatz (1) and thus generalize only the matter model. For this purpose it is convenient to write down the Einstein's equation for an arbitrary spherically symmetric energymomentum tensor $\boldsymbol{T}$. Recall that spherical symmetry implies for the component of $\boldsymbol{T}$ with respect to the orthonormal basis (10) that $\boldsymbol{T}\left(\boldsymbol{e}_{a}, \boldsymbol{e}_{A}\right)=0$ and $\boldsymbol{T}\left(\boldsymbol{e}_{A}, \boldsymbol{e}_{B}\right) \propto \delta_{A B}$, where $a \in\{0,1\}$ and $A, B \in\{2,3\}$. Hence, the only independent, non-vanishing components of $\boldsymbol{T}$ are

$$
\begin{aligned}
S & :=\boldsymbol{T}\left(\boldsymbol{e}_{0}, \boldsymbol{e}_{0}\right) \\
Q & :=\boldsymbol{T}\left(\boldsymbol{e}_{1}, \boldsymbol{e}_{1}\right) \\
P & :=\boldsymbol{T}\left(\boldsymbol{e}_{2}, \boldsymbol{e}_{2}\right) \\
J & :=-\boldsymbol{T}\left(\boldsymbol{e}_{0}, \boldsymbol{e}_{1}\right),
\end{aligned}
$$

and these are functions which do not depend on the angular coordinates. Note that $S$ is the energy density, $Q$ and $P$ the radial and spherical pressure, and $J$ the energy flow-all referred to the observer field $\boldsymbol{e}_{0}$. The sing in (31d) is chosen such that a positive $J$ means a flow of energy in positive radial direction. Taking (31) into account, the Einstein equation for the McVittie ansatz (11) and an arbitrary spherically symmetric energymomentum tensor $\boldsymbol{T}$ reduces to the following four equations:

$$
\begin{aligned}
& (a m)^{\cdot}=-4 \pi R^{2}\left(\frac{B}{A}\right)^{2} J \\
& 8 \pi S=3 F^{2} \\
& 8 \pi Q=-3 F^{2}-2 \dot{F}^{2} \frac{A}{B} \\
& P=Q .
\end{aligned}
$$

In view of (22), the first equation relates the time variation of the Weyl part of the MS energy contained in the sphere of radius $R$ with the energy flow out of it. The last equation is nothing but spatial Ricci-isotropy.

In the following subsections we will consider three models for the cosmological matter which generalize the original McVittie model: perfect fluid, perfect fluid plus heat flow, and perfect fluid plus null fluid.

\section{B. Perfect fluid}

Perhaps the simplest step one can take in trying to generalize the McVittie model is to stick to a single perfect fluid for the matter, but dropping the condition (3) of 'no-infall' by allowing for radial motions relative to the $\boldsymbol{\partial} / \boldsymbol{\partial} t$ observer field. In this way one could hope to avoid a particular singular behavior in the pressure that may be due to the 'no-infall' condition, though it is clear that the persisting geometric singularity must show up somehow in the matter variables as already discussed in Section IIID, Unfortunately, as already shown in [4], the relaxation of (3) does not lead to any new solutions.
What we want to stress here is that the reason for this, as shown in more detail below, lies precisely in the restriction imposed by spatial Ricci-isotropy.

We take thus the perfect-fluid energy-momentum tensor (2) for the matter and an arbitrary spherically symmetric four-velocity $\boldsymbol{u}$. The latter is given in terms of the orthonormal basis for the metric (11) by

$$
\boldsymbol{u}=\cosh \chi \boldsymbol{e}_{0}+\sinh \chi \boldsymbol{e}_{1},
$$

where $\chi$ is the rapidity of $\boldsymbol{u}$ with respect to the observer field $\boldsymbol{e}_{0}$ (a positive $\chi$ corresponds here to a boost in an outward-pointing radial direction). The non-vanishing components of the matter energy-momentum tensor (2) with four-velocity (33) are:

$$
\begin{aligned}
& \boldsymbol{T}\left(\boldsymbol{e}_{0}, \boldsymbol{e}_{0}\right)=\varrho+(\varrho+p) \sinh ^{2} \chi \\
& \boldsymbol{T}\left(\boldsymbol{e}_{0}, \boldsymbol{e}_{1}\right)=-(\varrho+p) \sinh \chi \cosh \chi \\
& \boldsymbol{T}\left(\boldsymbol{e}_{1}, \boldsymbol{e}_{1}\right)=p+(\varrho+p) \sinh ^{2} \chi \\
& \boldsymbol{T}\left(\boldsymbol{e}_{2}, \boldsymbol{e}_{2}\right)=\boldsymbol{T}\left(\boldsymbol{e}_{3}, \boldsymbol{e}_{3}\right)=p .
\end{aligned}
$$

Clearly, the case of vanishing rapidity must lead to the original McVittie model. In this case, in fact, the matter energy-momentum tensor (34) is already spatially isotropic so that (32d) is identically satisfied. Moreover, (32a) implies $(a m)^{\circ}=0$ and hence, in view of (14), $F=$ $\dot{a} / a$. Herewith Einstein's equation reduces to (4) and thus one gets back the original McVittie model.

In case of non-vanishing rapidity, spatial Ricciisotropy $(32 \mathrm{~d})$ implies the following constraint:

$$
\varrho+p=0 .
$$

This means that the energy momentum tensor (2) has the form of a cosmological constant (using (35) in $\operatorname{div} \boldsymbol{T}=0$ it implies $\boldsymbol{d} p=0$ and this, in turn, using again (35), implies $\boldsymbol{d} \varrho=0$ ) so that this case reduces to the Schwarzschild-de Sitter solution and hence does not provide the physical generalization originally hoped for.

\section{Perfect fluid plus heat flow}

In a next step one may keep (33) and drop the condition that the fluid be perfect, in the sense of allowing for radial heat conduction. This is described by a spatial vector field $\boldsymbol{q}$ that represents the current density of heat, which here corresponds to the current density of energy in the rest frame of the fluid. Hence $\boldsymbol{q}$ is everywhere orthogonal to $\boldsymbol{u} .^{7}$ The fluid's energy momentum tensor

\footnotetext{
7 We note that the parametrization of the energy-momentum tensor given in [4] is manifestly different. Whereas we parametrized it in the usual fashion in terms of quantities (energy density, pressure, current density of heat) that refer to the fluid's rest system, the authors of [4] also write down (36) (their Eq. (79)), but with $\boldsymbol{q}$ orthogonal to $\boldsymbol{e}_{0}$ (compare their Eq. (93)) rather than $\boldsymbol{u}$, which affects also the definition of $\varrho$. In fact, marking their quantities with a prime, their expression (79) is equivalent to our (36) iff $p=p^{\prime}, q=q^{\prime} \cosh \chi$, and $\varrho=\varrho^{\prime}-2 q^{\prime} \sinh \chi$.
} 
then reads

$$
\boldsymbol{T}=\varrho \underline{\boldsymbol{u}} \otimes \underline{\boldsymbol{u}}+p(\underline{\boldsymbol{u}} \otimes \underline{\boldsymbol{u}}-\boldsymbol{g})+\underline{\boldsymbol{u}} \otimes \underline{\boldsymbol{q}}+\underline{\boldsymbol{q}} \otimes \underline{\boldsymbol{u}} .
$$

Taking (33) as fluid velocity and imposing the heat flowvector $\boldsymbol{q}$ to be spherically symmetric, we have

$$
\boldsymbol{q}=q \boldsymbol{e}:=q\left(\sinh \chi \boldsymbol{e}_{0}+\cosh \chi \boldsymbol{e}_{1}\right),
$$

where $q$ is a function of $(t, r)$. Note that a positive $q$ corresponds to heat flowing in an outward-pointing radial direction. The independent non-vanishing components of the energy-momentum tensor are now as follows:

$$
\begin{aligned}
& \boldsymbol{T}\left(\boldsymbol{e}_{0}, \boldsymbol{e}_{0}\right)=\varrho+\tanh \chi((\varrho+p) \tanh \chi+2 q) \\
& \boldsymbol{T}\left(\boldsymbol{e}_{0}, \boldsymbol{e}_{1}\right)=q-\cosh ^{2} \chi((\varrho+p) \tanh \chi+2 q) \\
& \boldsymbol{T}\left(\boldsymbol{e}_{1}, \boldsymbol{e}_{1}\right)=p+\frac{1}{2} \sinh (2 \chi)((\varrho+p) \tanh \chi+2 q) \\
& \boldsymbol{T}\left(\boldsymbol{e}_{2}, \boldsymbol{e}_{2}\right)=\boldsymbol{T}\left(\boldsymbol{e}_{3}, \boldsymbol{e}_{3}\right)=p .
\end{aligned}
$$

Consider first the case of vanishing rapidity. Then the energy-momentum tensor is already spatially isotropic and Einstein's equation (32) reduces to

$$
\begin{aligned}
& (a m)^{\cdot}=-4 \pi R^{2} q\left(\frac{B}{A}\right)^{2} \\
& 8 \pi \varrho=3 F^{2} \\
& 8 \pi p=-3 F^{2}-2 \dot{F} \frac{A}{B} .
\end{aligned}
$$

These are three PDEs (though only time derivatives occur) for the five functions $a, m, \varrho, p$, and $q$ so that the system (39) is clearly under-determining. However, it is not possible to freely specify any two of these five functions and then determine the the other three via (39). For example, since the left-hand side of (39a) depends only on $t$, the same must hold for the r.h.s., which implies that $q=f(t) / r^{2}\left(1-(m / 2 r)^{2}\right)^{2}$, where $f(t)=-(a m)^{*} / 4 \pi a^{2}$. In particular, the heat flow must fall-off as $1 / r^{2}$.

The easiest way to generate a solution in the case of zero rapidity is to specify the two functions $a(t)$ and $m(t)$, then let $A, B, R, F$ be determined by the definitions (121314), and finally let the Einstein equations 39a/39b39c determine $q, \varrho$, and $p$, respectively. Notice that if we happen to specify $a$ and $m$ such that $a m$ is a constant, this immediately implies $q=0$ and $F=\dot{a} / a$, which leads to the standard McVittie solutions. From (39a) the following is evident: if $q>0(q<0)$, that is for outwardly (inwardly) pointing heat flow, the Weyl part of the MS energy decreases (increases), as one would expect.

Now we turn to the general case with non-vanishing rapidity: As it was the case for the perfect fluid in the previous subsection, the condition (32d) of spatial Ricciisotropy implies a constraint on the matter:

$$
(\varrho+p) \tanh \chi+2 q=0 .
$$

Using this, the other components of the Einstein's equation reduces to:

$$
\begin{aligned}
& (a m)^{\cdot}=+4 \pi R^{2} q\left(\frac{B}{A}\right)^{2} \\
& 8 \pi \varrho=3 F^{2} \\
& 8 \pi p=-3 F^{2}-2 \dot{F} \frac{A}{B} .
\end{aligned}
$$

These are almost the same as in the case of vanishing rapidity (see (39)), except for the opposite sign on the right-hand side of (40b). This simply results from the fact that, according to (38b), $J=-\boldsymbol{T}\left(\boldsymbol{e}_{0}, \boldsymbol{e}_{1}\right)=q$ for vanishing rapidity, whereas, due to the constraint (40a), $J=-\boldsymbol{T}\left(\boldsymbol{e}_{0}, \boldsymbol{e}_{1}\right)=-q$ for non-vanishing rapidity. This will be further interpreted below. Notice that for the equation of state $\varrho+p=0$ (cosmological term) (40a) implies $q=0$, thus leading once more to the Schwarzschildde Sitter solution (see comment below Eq. (35)). Henceforth we assume $\varrho+p \neq 0$, which implies that one can solve the constraint (40a) for the rapidity:

$$
\tanh \chi=-\frac{2 q}{\varrho+p}
$$

provided that $|2 q /(\varrho+p)|<1$.

The Einstein equation gives now four equations for the six functions $a, m, \varrho, p, q$, and $\chi$. As in the case of vanishing rapidity, this system is under-determining and it is not possible to freely specify any two of these six functions and then determine the the other four. In a similar fashion as before, the easiest way to generate a solution is to specify the two functions $a(t)$ and $m(t)$, to let then the definitions (1213 14) determine $A, B, R, F$, and finally use the Einstein equations (40b40c40d) and (41) to determine $q, \varrho, p$, and $\chi$, respectively. Again, choosing $a$ and $m$ such that their product is constant implies $q=0$ and $F=\dot{a} / a$, which leads to the standard McVittie solutions.

In passing we remark that the condition $\varrho+p>0$ can be expressed geometrically in terms of the second timederivative of the areal radius. Indeed, adding either (39b) to $39 \mathrm{c}$ or $40 \mathrm{c}$ to $40 \mathrm{~d}$ ) we obtain, taking into account $\boldsymbol{e}_{0}=(A / B) \boldsymbol{\partial} / \boldsymbol{\partial} t$ and (15):

$$
4 \pi(\varrho+p)=-\boldsymbol{e}_{0}\left(\frac{\boldsymbol{e}_{0}(R)}{R}\right),
$$

which is positive iff the rate of change $\boldsymbol{e}_{0}(R) / R$ is a decreasing function along the integral lines of the observer $e_{0}$. In other words, $\varrho+p$ is positive iff $\ln (R)$ is a concave function on the worldline of the observer $e_{0}$, which is implied by, but not equivalent to, the function $R$ being concave.

From (41) and (40b), and assuming $\varrho+p>0$, one sees the following: If $\chi>0(\chi<0)$, that is for an outwardly (inwardly) moving fluid with respect to $\boldsymbol{e}_{0}$, we have $q<0$ $(q>0)$, that is an inwardly (outwardly) pointing heat flow, and the Weyl part of the MS energy decreases (increases). This means that the heat flow's contribution to the change of $E_{\mathrm{W}}$ never compensates that of the fluid motion, quite in accord with naive expectation. Below we show that for small rapidities the contribution due to the heat flow is minus one-half that of the cosmological matter.

Let us now return to the sign-difference of the righthand sides of (39a) and (40b). From (38) one infers that $J$ is the sum of the two contributions coming from the 
heat flow

$$
J_{h}:=q\left(1+2 \sinh ^{2} \chi\right)
$$

and from cosmological matter

$$
J_{m}:=(\varrho+p) \sinh \chi \cosh \chi,
$$

respectively. The constraint (40a) can be written in the form

$$
2 \cosh ^{2} \chi J_{h}+\left(1+2 \sinh ^{2} \chi\right) J_{m}=0,
$$

which, for small rapidities $\chi$ (that is neglecting quadratic terms in $\chi$ ), implies $2 J_{h}+J_{m} \approx 0$. In this approximation the spatial energy-momentum flow due to heat is minus one-half that due to the cosmological matter. For the total flow this implies $J=J_{m}+J_{h} \approx J_{m} / 2 \approx-J_{h}$. Now the sign difference between (39a) and (40b) is understood as follows: In case of vanishing rapidity one has $J_{m}=0$, $J_{h}=q$ and hence $J=q$ (leading to (39a)), whereas a short calculation reveals that in case of non-vanishing rapidity the constraint (45) implies $J=J_{m}+J_{h}=-q$, leading thus to (40b).

\section{Perfect fluid plus null fluid}

The last tentative generalization we consider is taking for matter the incoherent sum (meaning that the respective energy-momentum tensors adds) of a perfect fluid (possibly with non-vanishing pressure) and a null fluid (eventually representing electromagnetic radiation). This clearly contains as special case the matter model considered by Sultana and Dyer [19] in which the pressure vanishes. We already stressed in Section III A that the metric ansatz of [19] is different from (11). Here we show that the matter model of [19] is essentially incompatible with (1) except for trivial or exotic cases.

The matter model consists of an ordinary perfect fluid and a null fluid (e.g. electromagnetic radiation) without mutual interaction. Hence the matter's energymomentum tensor is just the sum of (2) and

$$
\boldsymbol{T}_{\mathrm{nf}}^{ \pm}=\lambda^{2} \underline{\boldsymbol{l}}^{ \pm} \otimes \underline{\boldsymbol{l}}^{ \pm}
$$

where $\lambda$ is some non-negative function of $t$ and $r$ and $\boldsymbol{l}^{+}$and $\boldsymbol{l}^{-}$are, respectively, the outgoing and ingoing future-pointing null vector fields orthogonal to the spheres of constant radius $r$ partially normalized such that $\boldsymbol{g}\left(\boldsymbol{l}^{+}, \boldsymbol{l}^{-}\right)=1$. (It remains a freedom $\boldsymbol{l}^{ \pm} \mapsto \alpha^{ \pm 1} \boldsymbol{l}^{ \pm}$, where $\alpha$ is a positive function). Without loss of generality we make use of this freedom and choose:

$$
\boldsymbol{l}^{ \pm}=\left(\boldsymbol{e}_{0} \pm \boldsymbol{e}_{1}\right) / \sqrt{2}
$$

where $\boldsymbol{e}_{0}$ and $\boldsymbol{e}_{1}$ are the vectors of the orthonormal frame (10). The components of the whole energymomentum tensor with respect to this frame are then:

$$
\begin{aligned}
& \boldsymbol{T}\left(\boldsymbol{e}_{0}, \boldsymbol{e}_{0}\right)=\varrho+(\varrho+p) \sinh ^{2} \chi+\frac{1}{2} \lambda^{2} \\
& \boldsymbol{T}\left(\boldsymbol{e}_{0}, \boldsymbol{e}_{1}\right)=-(\varrho+p) \sinh \chi \cosh \chi \mp \frac{1}{2} \lambda^{2} \\
& \boldsymbol{T}\left(\boldsymbol{e}_{1}, \boldsymbol{e}_{1}\right)=p+(\varrho+p) \sinh ^{2} \chi+\frac{1}{2} \lambda^{2} \\
& \boldsymbol{T}\left(\boldsymbol{e}_{2}, \boldsymbol{e}_{2}\right)=\boldsymbol{T}\left(\boldsymbol{e}_{3}, \boldsymbol{e}_{3}\right)=p .
\end{aligned}
$$

Here and below the upper (lower) sign corresponds to the outgoing (ingoing) null field.

In the present case, the condition (32d) of spatial Ricciisotropy is equivalent to the constraint:

$$
(\varrho+p) \sinh ^{2} \chi+\frac{1}{2} \lambda^{2}=0 .
$$

In the physically relevant case in which $\varrho+p>0$ this equation has only the trivial solution $\chi=0$ and $\lambda=0$, which leads to the original McVittie model. In the case $\varrho+p=0$ (49) implies $\lambda=0$, leading thus to the Schwarzschild-deSitter spacetime (see comment below (35) ). Hence, a new solution is only possible if the matter is of an exotic type that satisfies $\varrho+p<0$, which either violates the weak energy-condition $(\varrho>0)$, or, less catastrophically, the dominant-energy condition $(\varrho>|p|)$. In particular, for the matter model considered by Sultana and Dyer, one would need to violate the weak energy-condition.

\section{CONCLUSION}

We conclude by commenting on the the main differences between these generalizations and the original McVittie model. First we stress once more that neither allowing for a nonzero rapidity nor a nonzero heat flow can eliminate the singularity at $r=m / 2(R=2 a m)$ (as erroneously stated in [4]). The only substantial new feature of these generalizations is that the Weyl part of the MS energy $E_{\mathrm{w}}=a m$ is not constant anymore. In view of the fact that the combination

$$
m / r=A^{2} E_{\mathrm{W}} / R \approx E_{\mathrm{W}} / R
$$

contained in the McVittie ansatz gives the 'Newtonian' part of the potential in the slow-motion and weak-field approximation (see [1]), we deduce that in order to get the geodesic equation for the generalized McVittie model, it suffices to substitute $m_{0}$ with $E_{\mathrm{w}}$ in the equation of motion derived in [1]. This means that the strength of the central attraction varies in time according to (32a), leading to an in- or out-spiraling of the orbits if $\boldsymbol{d} E_{\mathrm{W}}\left(\boldsymbol{e}_{0}\right)>0$ or $\boldsymbol{d} E_{\mathrm{W}}\left(\boldsymbol{e}_{0}\right)<0$, respectively.

We identified the origin of why we could not vary the rapidity and the heat flow independently in the condition (40a) of spatial Ricci-isotropy, which is built into the ansatz (11). We saw that this geometric feature renders this ansatz special, so that it would be improper to call it a general ansatz for spherical inhomogeneities in a flat FLRW universe. It remains to be seen whether useful generalizations exist which are captured by equally simple ansätze. 


\section{Acknowledgments}

D.G. acknowledges support from the Albert-EinsteinInstitute in Golm and the QUEST Excellence Cluster.

\section{APPENDIX A: PROOF OF PROPOSITION 1}

In this appendix we compute the intersection of the set $\mathcal{S}_{\mathrm{McV}}$ of metrics of type (11), which we denote in the following by $\boldsymbol{g}_{a, m}^{\mathrm{McV}}$, with the set $\mathcal{S}_{\mathrm{cS}}$ of metrics conformally related to an exterior Schwarzschild metric. Explicitly, the latter are of the form

$$
\boldsymbol{g}_{\Omega, M_{0}}^{\mathrm{cS}}:=\Omega^{2} \boldsymbol{g}_{M_{0}}^{\mathrm{Schw}},
$$

where

$$
\boldsymbol{g}_{M_{0}}^{\text {Schw }}=\left(1-\frac{2 M_{0}}{R}\right) \boldsymbol{d} T^{2}-\left(1-\frac{2 M_{0}}{R}\right)^{-1} \boldsymbol{d} R^{2}-R^{2} \boldsymbol{g}_{S^{2}},
$$

denotes the Schwarzschild metric with mass $M_{0}$ in 'standard' coordinates. The question is: for which functions $a$ and $m$ and, respectively, for which function $\Omega$ and parameter $M_{0}$ does the equation $\boldsymbol{g}_{a, m}^{\mathrm{McV}}=\Omega^{2} \boldsymbol{g}_{M_{0}}^{\mathrm{schw}}$ hold? Such an equality can be eventually established by finding a coordinate transformation, $\phi$ say, between the coordinates $^{8}(t, r)$ in (11) and $(T, R)$ in (A1) which brings (1) in form (A1). This involves solving coupled, non-linear partial differential equations for $\phi$, which depend on the four unknown parameter $a, m, \Omega$, and $M_{0}$. Needless to say that this is not really a thankful task. Alternatively, a better approach would be to compare all the independent, algebraic curvature-invariants of the two metrics: This would lead to a system of equations between scalars which involves the coordinate transformation $\phi$ in an algebraic way (i.e. non differentiated).

We adopt here an approach which is somewhere in the middle: First, we use just three invariants (the areal radius and the Ricci and the Weyl part of the MS energy) to drastically restrict the form of the coordinate transformation (see (A14) ) and derive thereby constraints on the free parameters $a, m, \Omega$, and $M_{0}$ (see (A10) and (A13)). Second, we perform this restricted coordinate transformation and determine it completely. To simplify the calculation, instead of $\boldsymbol{g}_{a, m}^{\mathrm{McV}}=\Omega^{2} \boldsymbol{g}_{M_{0}}^{\text {Schw }}$, we consider the equivalent equation $\Omega^{-2} \boldsymbol{g}_{a, m}^{\mathrm{McV}}=\boldsymbol{g}_{M_{0}}^{\text {Schw }}$. In fact, for the Schwarzschild metric (A1b) it is immediate that the above mentioned quantities are, respectively, given by:

$$
\begin{aligned}
& R\left(\boldsymbol{g}_{M_{0}}^{\text {Schw }}\right)=R, \\
& E_{\mathrm{W}}\left(\boldsymbol{g}_{M_{0}}^{\text {Schw }}\right)=M_{0}, \\
& E_{\mathrm{R}}\left(\boldsymbol{g}_{M_{0}}^{\text {Schw }}\right)=0 .
\end{aligned}
$$

\footnotetext{
8 The transformation between the angular variables is just the identity.
}

In order to compute the respective quantities for the metric $\Omega^{-2} \boldsymbol{g}_{a, m}^{\mathrm{McV}}$ we first give their scaling behavior under conformal transformations.

Clearly, because of their very definitions, for the areal radius and the Weyl part of the MS energy it holds:

$$
R\left(\Psi^{2} \boldsymbol{g}\right)=\Psi R(\boldsymbol{g})
$$

and

$$
E_{\mathrm{W}}\left(\Psi^{2} \boldsymbol{g}\right)=\Psi E_{\mathrm{W}}(\boldsymbol{g}),
$$

respectively. For the whole MS energy it easily follows from (20) and (A5):

$$
\begin{aligned}
E\left(\Psi^{2} \boldsymbol{g}\right)=\Psi & \left(E(\boldsymbol{g})+R^{2} \boldsymbol{g}(\boldsymbol{\nabla} R, \nabla \ln \Psi)\right. \\
& \left.+\frac{1}{2} R^{3} \boldsymbol{g}(\nabla \ln \Psi, \nabla \ln \Psi)\right),
\end{aligned}
$$

where all the quantities on the r.h.s. are referred to the metric $\boldsymbol{g}$. Hence, taking the difference between (A7) and (A6) one gets that the Ricci part of the MS energy scales exactly like the whole MS energy, that is according to (A7).

Using these scaling properties together with (13) and (22) we get immediately:

$$
\begin{aligned}
& R\left(\Omega^{-2} \boldsymbol{g}_{a, m}^{\mathrm{McV}}\right)=\Omega^{-1}(1+m / 2 r)^{2} a r \\
& E_{\mathrm{W}}\left(\Omega^{-2} \boldsymbol{g}_{a, m}^{\mathrm{McV}}\right)=\Omega^{-1} a m .
\end{aligned}
$$

The equality between the Weyl part of the MS energy (A9) and A3) implies

$$
\Omega(t, r) M_{0}=a(t) m(t),
$$

which gives a condition between the parameter $a, m, \Omega$, and $M_{0}$. Since we assumed that $M_{0}$ is positive, (A10) can be read as the expression for the conformal factor in the $(t, r)$ coordinates. This, together with the equality between the areal radius (A8) and (A2), implies in turn

$$
R(t, r)=\frac{M_{0}}{m(t)}(1+m(t) / 2 r)^{2} r,
$$

which gives the first component of the coordinate transformation $\phi$. Now, using the scaling property (A7) for the Ricci part of the MS energy, the expressions (21) and (13) for the Ricci part of the MS energy and, respectively, the areal radius of the McVittie metric ansatz, and (A10) for the conformal factor, one gets, after some computations,

$$
E_{\mathrm{R}}\left(\Omega^{-2} \boldsymbol{g}_{a, m}^{\mathrm{McV}}\right)=\frac{M_{0}}{2 a m}\left(A^{2} a r\right)^{3}\left(\frac{\dot{m}}{m}\right)^{2} .
$$

The equality between (A12) and (A4) then implies

$$
\dot{m}=0,
$$

that is $m=m_{0}$ for some positive constant $m_{0}$. This, in turns, implies that the transformation (A11) for $R$ depends only on $r$ and not on $t$. Since the metrics are both 
in diagonal form, this implies that the transformation for $T$ must depend on $t$ only.

Summarizing, so far we have seen that a set of necessary conditions for the equality of the two metrics implies the constraints (A10) and A13) and that the coordinate transformation between $(t, r)$ and $(T, R)$ is of the form

$$
\begin{aligned}
& T(t)=f(t) \\
& R(r)=\frac{M_{0}}{m_{0}}\left(1+m_{0} / 2 r\right)^{2} r,
\end{aligned}
$$

for some differentiable function $f$ of $t$. Now, explicitly expressing the metric $\Omega^{2} \boldsymbol{g}_{M_{0}}^{\text {Schw }}$ in the $(t, r)$ coordinates according to the coordinate transformation (A14) and the constraints (A10) and (A13), and putting the result equal to $\boldsymbol{g}_{a, m}^{\mathrm{McV}}$, the only new condition that one gets is

$$
\dot{f}= \pm \frac{M_{0}}{m_{0}} \frac{1}{a}
$$

Here, the plus can be chosen in order to exclude a time inversion. It is important to note that (together with an initial value) determines $f$ uniquely and do not give any constraint on the parameters $a, m, \Omega$, and $M_{0}$ : The only constraints remain thus (A10) and (A13).

The proof is concluded noticing that (A10) means that the only constraint on $\Omega$ is that, expressed in the $(t, r)$ coordinates, it depends on $t$ only and hence, in view of A14a and expressed in the $(T, R)$ coordinates, that it depends on $T$ only. More geometrically, this can be restated saying that the gradient of $\Omega$ must be proportional to $\boldsymbol{\partial} / \boldsymbol{\partial T}$, the Killing field of the Schwarzschild metric (see (A1b)).

\section{APPENDIX B: PROOF OF PROPOSITION 2}

Inserting the definition (14) of $F$ in the expression (23) for the Ricci scalar and organizing the result in powers of $r B \equiv r-m / 2$ we get:

$$
\begin{aligned}
\text { Scal }= & -12\left(\frac{\dot{a}}{a}\right)^{2} \\
& -\frac{6}{r B}\left(4 \frac{\dot{a}(a m)}{a^{2}}+r A\left(\frac{\dot{a}}{a}\right)\right) \\
& -\frac{6}{(r B)^{2}}\left(2\left(\frac{(a m)}{a}\right)^{\cdot}+r A\left(\frac{(a m)^{*}}{a}-\frac{\dot{a}(a m)}{a^{2}}\right)\right) \\
& -\frac{3}{(r B)^{3}} r A \frac{\dot{m}(a m)}{a} .
\end{aligned}
$$

Hence, the Ricci scalar remains finite in the limit $r \rightarrow$ $m / 2$ iff all the three coefficient of $(r B)^{-k}$, for $k \in$
$\{1,2,3\}$, vanish in this limit, that is iff it holds:

$$
\begin{aligned}
& 4 \frac{\dot{a}(a m)}{a^{2}}+m\left(\frac{\dot{a}}{a}\right)^{\cdot}=0 \\
& 2\left(\frac{(a m)}{a}\right)^{2}+m\left(\frac{(a m)}{a}-\frac{\dot{a}(a m)}{a^{2}}\right)=0 \\
& \dot{m}(a m)=0 .
\end{aligned}
$$

These conditions are clearly understood to hold for all times $t$ in which the functions $a$ and $m$ and their derivative exist. In view of (B2C) we have to distinguish between two cases: $\dot{m}=0$ and $(a m)^{\circ}=0$, respectively. In the first case the system (B2) reduces to the set of conditions $m\left(\ddot{a} / a+3(\dot{a} / a)^{2}\right)=0$ and $m\left(\ddot{a} / a+(\dot{a} / a)^{2}\right)=0$, which, in turn, reduces to $m=0$ (and $a$ arbitrary), corresponding to the FLRW metric, or to $\dot{a}=0$ (and $\dot{m}=0$ ), corresponding to the Schwarzschild metric. In the second case, in which $(a m)^{\cdot}=0,(\mathrm{~B} 2)$ reduces to $m(\dot{a} / a)^{\cdot}=0$, which implies either $m=0$ (and $a$ arbitrary), corresponding again to the FLRW metric, or $(\dot{a} / a)^{\circ}=0$. Together with $(a m)^{\circ}=0$, the latter corresponds to a McVittie metric with exponentially-growing (or -falling) scale factor $a(t)$, that is to a Schwarzschild-deSitter metric.

\section{APPENDIX C: SHEAR-FREE OBSERVER FIELDS IN SPHERICALLY SYMMETRIC SPACETIMES}

Towards the end of Section[I] we made use of the following result: A spherically symmetric normalized timelike vector field $\boldsymbol{u}$ in a spherically symmetric spacetime $(\mathcal{M}, \boldsymbol{g})$ is shear free iff the metric $\boldsymbol{h}_{\boldsymbol{u}}$ that $\boldsymbol{g}$ induces on the subbundle $\boldsymbol{u}^{\perp}:=\{\boldsymbol{v} \in T \mathcal{M} \mid \boldsymbol{g}(\boldsymbol{v}, \boldsymbol{u})=0\}$ by restriction is conformally flat.

To prove this, we first note that the subbundle $\boldsymbol{u}^{\perp}$ is integrable, in other words, $\boldsymbol{u}$ is hypersurface orthogonal. This follows from the spherical symmetry of $\boldsymbol{u}$, which implies that $\boldsymbol{u}^{\perp}$ contains the vectors tangent to the 2 dimensional $S O(3)$ orbits. Hence $\boldsymbol{u}$ essentially lives in the 2-dimensional orbit space $^{9}$, where it is trivially hypersurface orthogonal. The hypersurfaces orthogonal to $\boldsymbol{u}$ in 4-dimensional spacetime are then the preimages under the natural projection of the hypersurfaces (curves) in the 2-dimensional orbit space.

As a result, we may now locally introduce so-called isochronous comoving coordinates, with respect to which $\boldsymbol{u}=A(t, r)^{-1} \boldsymbol{\partial} / \boldsymbol{\partial} t$ and

$$
\boldsymbol{g}=A^{2}(t, r) \boldsymbol{d} t^{2}-B^{2}(t, r) \boldsymbol{d} r^{2}-R^{2}(t, r) \boldsymbol{g}_{S^{2}} .
$$

\footnotetext{
${ }^{9}$ The orbit space is the quotient $\mathcal{M} / \sim$, where $\sim$ is the equivalence relation whose equivalence classes are the orbits. It is a manifold on the subset corresponding to 2-sphere orbits, to which we restrict attention here. To say that " $\boldsymbol{u}$ essentially lives in the orbit space" means that $\underline{\boldsymbol{u}}$ is the pull-back of a 1 -form on the quotient via the natural projection.
} 
(Note the different meanings of the functions $A$ and $B$ as compared to (16)). We now consider the tangent-space endomorphisms $\boldsymbol{\nabla} \boldsymbol{u}: \boldsymbol{X} \mapsto \boldsymbol{\nabla}_{\boldsymbol{X}} \boldsymbol{u}$ and their projection into the orthogonal complement of $\boldsymbol{u}$, i.e.,

$$
\boldsymbol{\nabla}^{\perp} \boldsymbol{u}:=\left.\left(\boldsymbol{P}_{\boldsymbol{u}}^{\perp} \circ \nabla \boldsymbol{u} \circ \boldsymbol{P}_{\boldsymbol{u}}^{\perp}\right)\right|_{\boldsymbol{u}^{\perp}},
$$

where $\boldsymbol{P}_{\boldsymbol{u}}^{\perp}:=\mathbf{i d}-\boldsymbol{u} \otimes \underline{\boldsymbol{u}}$ is the projection orthogonal to $\boldsymbol{u}$ (id is the identity endomorphism in the tangent spaces of $\mathcal{M})$. Note that $\nabla^{\perp} \boldsymbol{u}$ is symmetric due to the hypersurface orthogonality of $\boldsymbol{u}$. A direct computation using (C1) yields

$$
\boldsymbol{\nabla}^{\perp} \boldsymbol{u}=\boldsymbol{u}(\ln (B)) \boldsymbol{P}_{r}+\boldsymbol{u}(\ln (R)) \boldsymbol{P}_{S^{2}},
$$

where $\boldsymbol{P}_{r}$ and $\boldsymbol{P}_{S^{2}}$ are the projections parallel to $\boldsymbol{\partial} / \boldsymbol{\partial} r$ and parallel to the tangent 2-planes to the $S^{2}$-orbits, respectively. The trace $\theta$ of $\nabla^{\perp} \boldsymbol{u}$, which gives the expansion of $\boldsymbol{u}$, is $\theta=\boldsymbol{u}(\ln (B))+2 \boldsymbol{u}(\ln (R))$, so that the tracefree part of $\nabla^{\perp} \boldsymbol{u}$, known as the shear endomorphism $\boldsymbol{\sigma}$, is given by:

$$
\boldsymbol{\sigma}:=\nabla^{\perp} \boldsymbol{u}-\frac{1}{3} \theta \mathbf{i d}^{\perp}=\sigma\left(\boldsymbol{P}_{S^{2}}-2 \boldsymbol{P}_{r}\right),
$$

where $\sigma:=\frac{1}{3} \boldsymbol{u}(\ln (R / B))$ denotes the shear scalar (only defined in a spherically-symmetric setting) and $\mathbf{i d}^{\perp}=$ $\boldsymbol{P}_{r}+\boldsymbol{P}_{S^{2}}$ the identity endomorphism in $\boldsymbol{u}^{\perp}$. In passing, we note that the defining equations for $\theta$ and $\sigma$ just given immediately lead to the following simple relation between the shear scalar, expansion, and the variation of the areal radius along $\boldsymbol{u}$, that we made use of in Section IIIB.

$$
\sigma+\theta / 3=\boldsymbol{u}(\ln (R))
$$

Now, according to (C4), the shear of $\boldsymbol{u}$ vanishes iff the shear scalar $\sigma$ does, that is, iff $\boldsymbol{u}(\ln (R / B))$ vanishes. This is equivalent to $R / B$ being independent of $t$ or to $R(t, r)=\mu(r) B(t, r)$ for some function $\mu$, so that the line element (C1) can be rewritten in the spatially conformally flat form

$$
\boldsymbol{g}=\tilde{A}^{2}(t, \rho) \boldsymbol{d} t^{2}-\tilde{C}^{2}(t, \rho)\left(\boldsymbol{d} \rho^{2}+\rho^{2} \boldsymbol{g}_{S^{2}}\right),
$$

where $\tilde{A}(t, \rho):=A(t, r(\rho)), \tilde{C}(t, \rho):=C(t, r(\rho))$, and

$$
C(t, r)=\frac{B(t, r) \mu(r)}{\rho(r)}
$$

with

$$
\rho(r)=\rho_{0} \exp \left\{\int_{r_{0}}^{r} \frac{d r^{\prime}}{\mu\left(r^{\prime}\right)}\right\}
$$

Hence we see that vanishing shear of $\boldsymbol{u}$ implies conformal flatness of the corresponding spatial metric. For the converse we first note that, since $\boldsymbol{u}$ and $\boldsymbol{g}$ are spherically symmetric, the spatial metric $\boldsymbol{h}_{\boldsymbol{u}}$ is itself spherically symmetric, so that $\boldsymbol{g}$ can be written in the form (C6a). This implies that the corresponding $R / B$ depends only on the radial coordinate and hence that the shear of $\boldsymbol{u}$ vanishes.
[1] Carrera, M., and D. Giulini, 2008, "On the influence of global cosmological expansion on the dynamics and kinematics of local systems," to appear in Reviews of Modern Physics, arXiv:0810.2712.

[2] Faraoni, V., 2009, "An analysis of the Sultana-Dyer cosmological black hole solution of the Einstein equations," arXiv:0907.4473.

[3] Faraoni, V., C. Gao, X. Chen, and Y.-G. Shen, 2009, "What is the fate of a black hole embedded in an expanding universe?," Physics Letters B 671, 7-9.

[4] Faraoni, V., and A. Jacques, 2007, "Cosmological Expansion and Local Physics," Physical Review D 76, 063510 (pages 16).

[5] Ferraris, M., M. Francaviglia, and A. Spallicci, 1996, "Associated radius, energy and pressure of McVittie's metric, in its astrophysical application," Nuovo Cimento B111, 1031-1036.

[6] Gao, C., X. Chen, V. Faraoni, and Y.-G. Shen, 2008, "Does the mass of a black hole decrease due to the accretion of phantom energy," Physical Review D D78, 024008 (pages 11).

[7] Gautreau, R., 1984, "Imbedding a Schwarzschild mass into cosmology," Physical Review D 29, 198-206.

[8] Hawking, S. W., 1968, "Gravitational Radiation in an Expanding Universe," Journal of Mathematical Physics 9(4), 598-604.

[9] Hernandez, J., Walter C., and C. W. Misner, 1966, "Ob- server Time as a Coordinate in Relativistic Spherical Hydrodynamics," Astrophysical Journal 143, 452-464.

[10] Klioner, S. A., and M. H. Soffel, 2005, "Refining the Relativistic Model for Gaia: Cosmological Effects in the BCRS," in The Three-Dimensional Universe with Gaia, edited by C. Turon, K. S. O'Flaherty, and M. A. C. Perryman (ESA), volume 576 of ESA Special Publication, 305-308, proceedings of the Symposium "The Three-Dimensional Universe with Gaia", 47 October 2004, Observatoire de Paris-Meudon, France, arXiv:astro-ph/0411363.

[11] Komatsu, E., et al., 2009, "Five-Year Wilkinson Microwave Anisotropy Probe (WMAP) Observations: Cosmological Interpretation," Astrophysical Journal, Supplement Series 180, 330-376.

[12] McVittie, G. C., 1933, "The mass-particle in an expanding universe," Monthly Notices of the Royal Astronomical Society 93, 325-339.

[13] Misner, C. W., and D. H. Sharp, 1964, "Relativistic Equations for Adiabatic, Spherically Symmetric Gravitational Collapse," Physical Review 136, 571-576.

[14] Nolan, B. C., 1993, "Sources for McVittie's Mass Particle in an Expanding Universe," Journal of Mathematical Physics 34(1), 178-185.

[15] Nolan, B. C., 1998, "A point mass in an isotropic universe: Existence, uniqueness, and basic properties," Physical Review D 58(6), 064006 (pages 10). 
[16] Nolan, B. C., 1999, "A point mass in an isotropic universe: II. Global properties," Classical and Quantum Gravity 16, 1227-1254.

[17] Nolan, B. C., 1999, "A point mass in an isotropic universe: III. The region $R \leq 2 m$," Classical and Quantum Gravity 16, 3183-3191.

[18] Robertson, H. P., 1928, "On Relativistic Cosmology," Philosophical Magazine 5, 835-848.
[19] Sultana, J., and C. C. Dyer, 2005, "Cosmological black holes: A black hole in the Einstein-de Sitter universe," General Relativity and Gravitation 37(8), 1349-1370.

[20] Sussman, R. A., 1988, "On spherically symmetric shearfree perfect fluid configurations (neutral and charged). III. Global view," Journal of Mathematical Physics 29(5), 1177-1211. 